\title{
La estrategia de trabajo colaborativo del proyecto UNA Educación de Calidad de la Universidad Nacional de Costa Rica: Experiencia de la modalidad de talleres
}

\section{Collaborative Work Strategy in the Universidad Nacional de Costa Rica-UNA Educación de Calidad Project: Workshop Experiences}

\author{
María Roxana Rodríguez Araya \\ Universidad Nacional \\ División de Educación Básica, Centro de Investigación y Docencia en Educación, \\ Heredia, Costa Rica \\ rodri156@gmail.com \\ Angélica Fontana Hernánde² \\ Universidad Nacional \\ División de Educación Básica, Centro de Investigación y Docencia en Educación, \\ Heredia, Costa Rica \\ angelfontana@ice.co.cr
}

\begin{abstract}
Recibido 31 de julio de 2013 • Corregido 19 de octubre de 2013 • Aceptado 30 de octubre de 2013
Resumen. Este artículo presenta las vivencias del grupo de estudiantes de la Universidad Nacional de Costa Rica con una condición de discapacidad o que presenta necesidades educativas en su formación profesional. De acuerdo con la experiencia generada desde el quehacer del proyecto UNA Educación de Calidad en el contexto universitario, en los años 2008 al 2012, el equipo de trabajo tomó la decisión de reflexionar sobre las formas de apoyo y el seguimiento a este grupo de la diversidad, específicamente valora las acciones del trabajo colaborativo realizado por el grupo de participantes en el proyecto mediante la modalidad de talleres durante este periodo. Para una mayor comprensión de la propuesta desarrollada, los diferentes talleres se agrupan por módulos según su temática. Los resultados muestran la complejidad y los matices de las acciones de colaboración entre el grupo de estudiantes con el fin de responder a la realidad educativa imperante. A raíz de los resultados se vislumbra la necesidad de que el equipo del proyecto continúe desplegando una metodología con un enfoque constructivista que permita al grupo de participantes continuar colaborando desde sus intereses, habilidades y destrezas a la comunidad universitaria.
\end{abstract}

Palabras claves. Trabajo colaborativo, aprendizaje significativo, diversidad, discapacidad, necesidades educativas, inclusión social.

Maestría Académica de la Universidad de Minnesota, certificado en Desórdenes del Espectro Autista de la Universidad de Minnesota. Bachiller en Educación Preescolar de la Universidad Estatal a Distancia de Costa Rica. Laboró como investigadora asociada en HACER (Hispanic Advocacy and Community Empowerment through Research) y centros educativos dentro y fuera del país. Actualmente labora como académica en la Universidad Nacional, en la División de Educación Básica y en el proyecto UNA Educación de Calidad.

2 Master en Pedagogía con énfasis en la Diversidad de Procesos Educativos. Licenciada en Educación Especial con énfasis en Retardo Mental y Bachiller en Problemas de Aprendizaje de la Universidad Nacional de Costa Rica. Académica de la División de Educación Básica del Centro de Investigación y Docencia en Educación (CIDE) de la Universidad Nacional, Costa Rica y responsable del proyecto UNA educación de calidad, que atiende estudiantes con necesidades educativas en su formación profesional. Con experiencia en educación primaria y en los servicios de educación especial. Tiene publicaciones en el campo de la pedagogía en el ámbito nacional e internacional. 


\begin{abstract}
This paper presents the experiences of a group of students with disabilities or educational needs in their professional development at the National University in Costa Rica. Based on the experience obtained in the Quality Education Project from Universidad Nacional (UNA) (UNA Educación de Calidad) between 2008 and 2012, the work team decided to focus on ways to support and follow up on this diversity group, particularly taking into consideration the collaborative work done by participants during the workshops for this period. To facilitate the project, the workshops were grouped into modules by topics. Results show the complexity and different shades of the collaborative actions between the groups of students in order to respond to the current educational reality. Based on the results, the need for the project team to continue developing this constructivist methodology is clear in order to allow participants to continue offering their contributions to the rest of the university community using their own interests and skills.
\end{abstract}

Keywords. Collaborative work, significant learning, diversity, disabilities, educational needs, social inclusion.

Desde la segunda mitad del siglo XX, Costa Rica se encaminó a un cambio político, social y económico relacionado con la equiparación de derechos de las personas en condición de discapacidad. Estos esfuerzos se reflejaron en el ámbito nacional, con la promulgación de la Ley 7600 Igualdad de oportunidades para las personas con discapacidad (Costa Rica, Asamblea Legislativa, 1996) y la Ley 8661 Convención de los derechos de las personas con discapacidad (Costa Rica, Asamblea Legislativa, 2008), asimismo, con la Política nacional en materia de discapacidad -PONADIS- (Consejo Nacional de Rehabilitación y Educación Especial [CNREE], 2011), las cuales fueron influenciadas por convenios internacionales firmados por el país, con la meta de orientar y articular los esfuerzos a nivel de normativas públicas que velaran por los derechos de las personas con discapacidad.

Los esfuerzos de las voluntades nacionales e internacionales han promovido un cambio en la forma en que se visualiza y se contextualiza a la persona en condición de discapacidad. Es así como, actualmente se percibe como el resultado de la interacción de las personas que presentan deficiencias y las barreras en las actitudes de la sociedad que evitan la participación plena y efectiva en igualdad de condiciones (ONU, 2006).

La concepción social de la discapacidad ha promovido que, en los distintos foros internacionales y nacionales, las discusiones sobre la temática no se centren en las deficiencias que presentan las personas, sino, por el contrario, en las capacidades y en la promoción de espacios sociales para la inclusión social, educativa y económica de este grupo de la sociedad.

Estas leyes y políticas han impulsado cambios en la organización de las instituciones educativas, tanto en el ámbito administrativo como curricular. Por lo anterior, en el contexto universitario, desde el quehacer del proyecto UNA Educación de Calidad se proporciona apoyo y seguimiento a este grupo de la diversidad, cumpliendo, de esta forma, con los principios de justicia y solidaridad que norman el trabajo de la Universidad Nacional (Universidad Nacional [UNA], 1993). 
Dentro de esta perspectiva, la eliminación de las barreras para el acceso a la educación superior constituye una consigna en el quehacer del proyecto, particularmente para ofrecer al grupo de estudiantes participantes, matriculados en distintas carreras de la institución, una atención de calidad.

Por tanto, en la dinámica interna del proyecto, se concibe a cada uno de sus miembros desde una visión integral, con habilidades de autodeterminación e independencia, que les permiten no solo relacionarse e intercambiar sus conocimientos, habilidades y destrezas, sino reconstruirse interiormente, al reflexionar y replantear sus percepciones y construcciones de la realidad educativa.

Si bien estas acciones repercuten en la atención educativa que se ofrece desde los programas, los proyectos y los departamentos institucionales, continúan existiendo en el contexto universitario retos para atender la diversidad, específicamente al grupo que presenta una condición de discapacidad $u$ otras situaciones personales y sociales que les generan necesidades educativas.

En este sentido, Fontana (2009) identifica tres retos:

El primero es el reconocimiento del valor de la diversidad. Para responder a este desafío propone que debe existir un cambio en múltiples niveles, tanto en las estructuras actitudinales, cognitivas y emocionales del grupo de actores educativos, como en la organización administrativa y curricular en el ámbito institucional. Cuando se reconoce la diversidad como un valor, se permite percibir al ser humano desde una visión integral, con diferentes condiciones personales, sociales y culturales (donde la discapacidad es una característica más del abanico social), las cuales constituyen una oportunidad para el conglomerado, porque permite desplegar mayores destrezas y habilidades de interacción y convivencia.

El segundo reto es generar las condiciones para una vida universitaria en diversidad. Esto implica promover, en primera instancia, la accesibilidad al espacio físico y a la infraestructura; asimismo, generar los apoyos requeridos por la población estudiantil con el propósito de que pueda acceder a los servicios ofrecidos por los distintos departamentos y programas institucionales. También, involucra la creación de espacios para la discusión y reflexión sobre los valores para la convivencia en diversidad, encabezados por la tolerancia, el respeto, la solidaridad, la justicia y la responsabilidad social; asimismo, para la construcción de las diversas identidades de los grupos que conforman la población estudiantil y su inclusión social en la comunidad nacional.

Finalmente, el tercer reto consiste en construir un currículo para la diversidad que responda a los principios de igualdad y equidad de oportunidades para toda la población estudiantil, con propuestas pedagógicas y metodológicas incluyentes, particularmente, del grupo que presenta una condición de discapacidad o necesidades educativas. Para cumplir este cometido, se requiere visualizar el currículo de forma flexible y amplia, de manera que admita la construcción y reconstrucción de conocimientos para la vida en comunidad, de la mano con los objetivos y contenidos específicos de cada disciplina o especialidad. 
URL: http://www.una.ac.cr/educare

CORREO: educare@una.cr

Considerando los retos para la atención de la diversidad, particularmente del grupo en condición de discapacidad o que presenta necesidades educativas en el contexto universitario y aunado a la visión social emergente, que resalta el reconocimiento de sus capacidades, es necesario identificar los apoyos que ofrece el proyecto UNA Educación de Calidad dentro de la estructura universitaria, así como los aportes de este grupo a la comunidad educativa.

Por lo anterior, este artículo tiene el propósito de reflexionar sobre las formas de apoyo y el seguimiento desplegadas desde el marco de este proyecto, específicamente valora las acciones del trabajo colaborativo realizado por el grupo de participantes mediante la modalidad de talleres durante los años 2008 al 2012. Estos esfuerzos, tanto del equipo del proyecto como del grupo de estudiantes, se visualizan dentro de las acciones de cooperación promotoras de un crecimiento personal y social para ambos actores.

\section{El proyecto UNA Educación de Calidad en el contexto de la Universidad Nacional}

El proyecto UNA Educación de Calidad código 0499-11 (Fontana y Rodríguez, 2011) emergió en el año 1998 como respuesta a la realidad en la que estaba inmerso el sistema educativo nacional, particularmente, en el ámbito universitario. La propuesta pedagógica desplegada da muestra de una transición entre el modelo con un enfoque clínico- rehabilitador a un modelo social. Dentro de cada uno de estos, es posible apreciar el respectivo abordaje educativo, que va desde los procedimientos de la educación integradora a la educación inclusiva.

Desdeesta perspectiva, el proyectoemprendela tarea de proporcionarapoyoyseguimiento en su formación profesional a un grupo de la diversidad, como lo es el grupo de estudiantes que presenta una condición de discapacidad o necesidades educativas matriculados en las distintas carreras de la Universidad Nacional y, de esta forma, hacer efectivos sus derechos de acceder a una educación superior de calidad, de acuerdo con sus características personales y sociales, promoviendo de esta forma, su inserción social y laboral.

Los retos que impone la atención a la diversidad en el contexto universitario son un desafío que se plasma en el quehacer cotidiano del proyecto. Estas transformaciones se vislumbran en la constante reconstrucción de las estrategias de apoyo empleadas para responder a las necesidades educativas del grupo de estudiantes participantes, así como en el establecimiento de nuevas formas de trabajo colaborativo entre los miembros de la comunidad universitaria.

\section{La redes de apoyo: Una estrategia desplegada por el proyecto UNA Educación de Calidad}

La dinámica interna del proyecto responde al modelo social emergente y plasma, en su accionar, una serie de actividades que promueven la independencia del grupo de estudiantes participantes, con especial atención al que presenta una condición de discapacidad o necesidades educativas, a través de los principios de autodeterminación y vida independiente. 
El principio de vida independiente nace en la década de los 70 en los Estados Unidos, como producto de los esfuerzos de los grupos y organizaciones de personas con discapacidad que abogaban por el reconocimiento de sus derechos individuales y sociales, específicamente, por llevar una vida autónoma, es decir, ser quienes toman la dirección de sus propias vidas. En este sentido, Abad, Álvarez, y Castro (2008) resaltan que este movimiento plasma el derecho de este grupo de la diversidad a acceder, en igualdad de condiciones, a las mismas situaciones de vida y a tomar sus propias decisiones, según sus condiciones personales y sociales.

El proyecto, por su complejidad y su propia sinergia, crea redes de apoyo entre los diferentes actores que lo conforman; asimismo, por la visión constructivista que rige su accionar, ha generado que las formas de apoyo se interconecten promoviendo la discusión y el intercambio de experiencias que enriquecen tanto las dinámicas sociales, como los conocimientos construidos que aportan al crecimiento profesional del personal académico y administrativo y, especialmente, de la población estudiantil.

Entre las principales acciones de apoyo que se desarrollan en la dinámica interna del proyecto se distinguen las siguientes:

- Inducción al grupo de estudiantes de nuevo ingreso que presenta una condición de discapacidad o necesidades educativas que se incorpora cada año a la UNA. En primera instancia, se coordina con la Unidad de Admisión del Departamento de Registro para identificar la población admitida en alguna carrera de la institución en el I ciclo lectivo. Posteriormente, se contacta a este grupo de estudiantes para realizar una entrevista inicial con el fin de presentar los objetivos del proyecto, registrar los datos personales, determinar las necesidades educativas, los apoyos y el seguimiento que requieren.

- Apoyo en el estudio al grupo de participantes. A través de la experiencia se ha evidenciado que existe un grupo de estudiantes que necesitan apoyo específico en diferentes áreas, como por ejemplo en matemáticas, español, específicamente en la redacción y ortografía, en indagación bibliográfica e inglés. El equipo del proyecto promueve la colaboración de un grupo de compañeros avanzados de las carreras de pedagogía, quienes dan apoyo individual al grupo de estudiantes que presentan necesidades específicas, con lo cual se han obtenido resultados muy positivos.

- Apoyo técnico para estudiantes con discapacidad visual. El equipo del proyecto ofrece ayudas técnicas a este grupo de estudiantes, por medio de préstamo de recursos tecnológicos como grabadoras digitales y computadoras portátiles y la instalación de programas especializados. También, promueve el aprendizaje entre pares, para la utilización del programa lector de pantalla JAWS. Esta colaboración entre un estudiante 
que conoce el programa y otro que requiere utilizarlo ha resultado muy efectiva en el ámbito social y académico, ya que favorece tanto el intercambio de conocimientos como de experiencia personales y sociales; asimismo se crean lazos de amistad perdurables en sus formación profesional.

- Apoyo directo al grupo de estudiantes. Los miembros de trabajo del proyecto propician la atención de las necesidades educativas del grupo participante, a través de la coordinación con las diferentes unidades académicas en que se encuentran inscritos. En situaciones específicas, especialmente en condición de discapacidad, se realiza un seguimiento de los cursos por medio de la asesoría al personal académico y se efectúan visitas a los salones de clase, cuando así se amerita. También, se ofrece un espacio de dialogo para que el grupo de estudiantes pueda conversar sobre sus inquietudes, necesidades personales u otras situaciones vividas en el contexto universitario. En este sentido, cuando se requiere, se realizan referencias al Departamento de Orientación y Psicología, al de Salud o al de Bienestar Estudiantil para que reciban el apoyo según los servicios.

- Asesoría y capacitación al personal académico y administrativo. Como una acción acertada, se realizan sesiones de asesoría y capacitación al personal académico y administrativo de las distintas unidades académicas en que se encuentra inscrito el grupo de participantes. Anualmente se atiende, aproximadamente, a 150 académicos con el fin de conversar sobre las formas de atención pedagógicas a las necesidades educativas, los ajustes metodológicos y evaluación. En cada asesoría se completa un documento con los puntos tratados y los acuerdos realizados.

- Elaboración de materiales informativos y de apoyo a la docencia. Tomando en consideración la experiencia acumulada durante 15 años, el equipo del proyecto ha realizado material didáctico sobre la atención a las necesidades educativas de la población con discapacidad y con otras condiciones personales y sociales, tales como desplegables informativos y guías de apoyo para el personal académico que tiene inscritos en los cursos que imparte estudiantes con discapacidad visual.

- Promoción de la accesibilidad de la información y la comunicación. Se coordina con el personal académico y el grupo de estudiantes con discapacidad visual el proceso de digitalización del material que se requiere en los cursos, así como la impresión de diferentes materiales en el sistema braille, en relieve o ampliado. Este apoyo se realiza especialmente en los cursos de matemática, estadística, contabilidad, inglés u otras materias que requieran un material adaptado.

- Fomento de la accesibilidad al espacio físico y a la infraestructura. Se coordina, con el área de Planeamiento Espacial de la institución o las unidades académicas, la construcción de las adaptaciones o ajustes en este ámbito, con el fin de garantizar la participación plena del grupo de estudiantes con discapacidad. 
- Coordinación con otras instancias universitarias. Se coordina con los distintos departamentos, programas y proyectos de la institución para responder a los requerimientos del grupo de participantes, particularmente se realizan referencias al Programa de Éxito Académico para la asignación de tutores en el área de la matemática e inglés y al Departamento de Orientación y Psicología para el apoyo y seguimiento en psicología, valoraciones en psicopedagogía y orientación vocacional (Fontana y Rodríguez, 2012).

\section{Mirando el trabajo colaborativo desde la experiencia de los talleres en el proyecto UNA Educación de Calidad}

Desde el enfoque social, se otorga un reconocimiento a la diversidad de formas de aprender del ser humano. Por esto surge la necesidad de reflexionar en el contexto educativo, en general, particularmente en el universitario, sobre dos elementos de importancia.

En primera instancia, la Universidad Nacional, por su función social, debe convertirse en un espacio que propicie y reconozca la heterogeneidad en la forma en que el ser humano aprende y construye el conocimiento, específicamente, debe promover el desarrollo de destrezas y habilidades que le permitan a la población estudiantil participar activamente en la propuesta de ideas para resolver situaciones en el ámbito educativo y social, propiciando de esta forma su empoderamiento y transformación.

Como segundo elemento, existe la necesidad de mirar la colaboración como un aspecto dinamizador que promueve el aprendizaje entre el grupo de participantes. En el contexto universitario, se destaca los aportes del grupo de estudiantes, del personal docente y administrativo de las unidades académicas; asimismo del personal de los distintos departamentos, programas, proyectos de la institución.

Cuando la colaboración se visualiza dentro de la dinámica del quehacer universitario, se trasciende el plano meramente académico y se prepara a la comunidad estudiantil para participar en una sociedad que demanda, cada vez más, las destrezas de comunicación asertiva y el trabajo en equipo.

En esta línea, Maldonado y Sánchez (2012) mencionan que la colaboración conlleva tres elementos indispensables: la interactividad de quienes colaboran en un ambiente de intercambio horizontal; la sincronía de la interacción, al asumir una posición hacía el logro común de un objetivo, y la negociación como un proceso continuo. Más aún, los autores advierten que la colaboración "constituye un proceso esencialmente humano que exige el desarrollo de destrezas sociales y demanda un accionar concertado" (pp. 103-104).

El trabajo concertado entre las diversas instancias universitarias para promover la inserción social y educativa del grupo de estudiantes con discapacidad o que presentan necesidades educativas ciertamente es un desafío. Sin embargo, son evidentes los beneficios de promover 
URL: http://www.una.ac.cr/educare

CORREO: educare@una.cr

el trabajo colaborativo en la comunidad universitaria. Entre estos resalta el desarrollo de competencias sociales adquiridas en el proceso colaborativo, las cuales le permiten al grupo de participantes (estudiantes, académicos, administrativos) acercarse al aprendizaje de una forma más consciente y libre.

En este contexto, la colaboración se visualizará como un proceso que promueve aprendizajes significativos, pues se logra comprender el objetivo y la funcionalidad de lo que se está aprendiendo. Es decir, propicia que el grupo de participantes relacione sus experiencias de vida con la cotidianidad educativa facilitando, especialmente a la población estudiantil, un crecimiento en el área personal y social que promoverá su futura inserción en el mundo laboral.

Los principios de funcionalidad y significatividad del aprendizaje a través de la colaboración se despliegan en la modalidad de talleres, ya que su dinámica interna permite la reconstrucción personal y grupal de las experiencias de vida.

Los talleres como estrategia pedagógica se fundamentan en tres ejes, a saber: El primero se refiere a que esta modalidad ofrece un espacio para la atención de las necesidades específicas del grupo de participantes, es decir, las temáticas abordadas en los talleres siempre deben ser de interés para los involucrados. El segundo eje alude a la importancia de la participación en el desarrollo de las actividades; esto, por cuanto, al ofrecer los talleres un proceso de interacción e intercambio de experiencias y de conocimientos, se incrementa la motivación, el sentimiento de logro, la empatía y la colaboración por parte del grupo de participantes en un aprendizaje significativo. Finalmente, el tercero es la visualización, en la cual se combinan los dos aspectos anteriores. Es así como el intercambio que se realiza en los talleres promueve, a su vez, que se satisfagan las necesidades específicas de quienes participan (Candelo, Ortiz y Unger, 2003).

En relación con el empleo de los talleres en el contexto educativo, Cortes e Iriarte (s. f.), resaltan que el grupo de estudiantes logra aprendizajes significativos con actividades que les permiten:

- Participar activamente.

- Relacionar lo que tiene que aprender con lo que ya sabe.

- Participar en actividades diversificadas, adaptables y variadas, en situaciones y contextos reales o lo más cercano posible a lo real.

- [Trabajar] con materiales funcionales, acordes con su edad, su cultura..., percibiendo un sentido y utilidad práctica en lo que aprende.

- Obtener los apoyos y ayudas que precisa respetado su ritmo y estilo de aprendizaje.

- Sentir el reconocimiento al esfuerzo y al trabajo que desarrolla. (p. 2) 
También, Echeita y Martín (1999) aportan en este sentido. Indican que en la consecución de aprendizajes significativos se involucran tanto proceso cognitivos como de tipo motivacional, socio afectivo o relacional en un sentido amplio (aceptación- rechazo; cariño-empatía; igualdadsumisión), los cuales, mediatizados por estrategias pedagógicas incluyentes, como la modalidad de talleres, alcanzan las metas comunes.

Los principios anteriormente mencionados se consideran en las distintas modalidades de los talleres que se despliegan en el proyecto UNA Educación de Calidad, ya que estos se desarrollan como una estrategia de trabajo colaborativo que promueve un intercambio de experiencias basadas en las vivencias personales y en los procesos educativos del grupo de participantes; asimismo, permite la reflexión interior que se contextualiza dentro de las historias de vida, facilitando el desarrollo de competencias personales y una reelaboración de la experiencia de vida.

\section{Metodología}

El proyecto UNA Educación de Calidad se circunscribe, en la II fase de formulación 2012 al 2014, en el enfoque cualitativo y responde al tipo de investigación acción, debido a que su accionar se construye como un proceso dinámico y dinamizador generado por el grupo involucrado (Fontana y Rodríguez, 2011).

Desde esta perspectiva, el equipo del proyecto despliega una estrategia de trabajo colaborativo mediante la modalidad de los talleres. De acuerdo con Hernández, Fernández y Batista (2006), esta técnica de trabajos en grupo se utiliza para lograr, con eficacia, metas comunes, lo cual permite integrar la actuación del grupo de participantes en diferentes actividades, tanto en forma individual como colectiva, y en diferentes momentos.

Considerando lo anterior, se realizó una serie de reuniones y entrevistas (no estructuradas con cinco preguntas abiertas) con el grupo de participantes, para determinar los requerimientos en el ámbito personal, académico y social. Con la información recabada se construyó una matriz según la coincidencia y la divergencia entre los aspectos mencionados para identificar las áreas prioritarias para desplegar en el abordaje.

Es importante indicar que cada uno de los talleres surge como respuesta de las necesidades detectas en el quehacer cotidiano del proyecto durante los años 2008 al 2012, asimismo del perfil (capacidades y formación profesional) de los miembros del equipo de trabajo de cada año. Por estas razones, se presentan, a continuación, las características generales del proceso seguido en la construcción de las distintas modalidades de talleres. No obstante, en la elaboración de cada taller se requirió desplegar variadas acciones generadas por el tipo de temática que se abordaba, las particularidades del grupo de participantes, el tiempo y los recursos disponibles. 
URL: http://www.una.ac.cr/educare

CORREO: educare@una.cr

En forma general, se realizó, en primera instancia, un conversatorio con el grupo de estudiantes que realizaba labores de asistencia en el proyecto, particularmente con los estudiantes que presentaban una condición de discapacidad visual, con el fin de conocer sus ideas, inquietudes en este sentido $y$, especialmente, para identificar los aportes que podría proporcionar desde sus ámbitos de formación (orientación, psicología, educación especial, pedagogía en I y II ciclos y educación preescolar).

En segundo lugar, se efectuaron conversatorios con el grupo de estudiantes que participaban del proyecto para saber, en forma puntual, cuáles eran los requerimientos inmediatos para su desarrollo personal y formación profesional; asimismo el tiempo disposición para integrarse en las distintas actividades.

En tercero lugar, el equipo se reunió para sistematizar la información recabada y establecer los requerimientos inmediatos del grupo de estudiantes participantes y las posibles acciones por desarrollar. En este sentido, se visualizó la modalidad de talleres como una estrategia de trabajo colaborativo muy enriquecedora, porque permite no solo atender las necesidades educativas que presenta el grupo de participantes, sino potenciar mayores destrezas y habilidades en el ámbito personal y social, según sus intereses y motivaciones. Los talleres se diseñaron como módulos

Los talleres se diseñaron como módulos.

\section{Participantes}

Los participantes de los talleres han sido muchos y variados, esto responde a la diversidad imperante en el proyecto y a la visión de inclusión que marca el accionar en el quehacer diario.

Durante el periodo del 2008 al 2012 se desarrollaron diversos talleres con la participación de 6 estudiantes que realizan labores de asistencia ${ }^{3}(\mathrm{HA})$ en el proyecto, dos de estas personas presentaban una condición de discapacidad visual; 38 estudiantes que realizaban labores de colaboración ${ }^{4}(\mathrm{HC}) ; 50$ estudiantes que presentan necesidades educativas o una condición de discapacidad; 30 del personal académico y más de 150 estudiantes de la comunidad universitaria de diversas carreras como Educación Especial, Enseñanza del Inglés, Pedagogía en I y II ciclo de la Educación General Básica, Psicología, Administración, Economía, Planificación y Promoción Social, entre otras.

3 Estudiante becado por la Universidad Nacional y avanzado en su carrera que realiza diferentes labores en la unidades académicas, departamentos, proyectos y programas con una retribución económica

$4 \quad$ Estudiante becado por la Universidad Nacional y avanzado en su carrera que realiza diferentes labores en las unidades académicas, departamentos, proyectos y programas sin una retribución económica. 


\section{Experiencias en los talleres dentro del proyecto UNA Educación de Calidad}

\section{Módulo 1: Talleres ofrecidos por estudiantes con discapacidad visual a la comunidad universitaria}

\section{Talleres de apoyos tecnológicos para personas con discapacidad visual}

Los talleres de apoyos tecnológicos para personas con discapacidad visual se ofrecen para la comunidad estudiantil desde el año 2010, a las distintas carreras de la universidad que lo soliciten, particularmente, se ha impartido en cursos de la carrera de Psicología, Pedagogía para I y ll ciclos del Educación Básica y Educación Especial con énfasis en Integración Educativa. Estos talleres fueron construidos por un estudiante con discapacidad visual que realizaba HA en el proyecto, con apoyo de las académicas encargadas. Durante el año 2010 los talleres fueron impartidos por este estudiante a la comunidad estudiantil $y$, posterior a su egreso de la universidad, otros estudiantes con discapacidad visual y estudiantes asistentes han apoyado este proceso.

Para una mayor comprensión se presenta en la tabla 1 la descripción de primer grupo de talleres de este módulo.

Tabla 1

Tema y objetivo de los talleres de apoyos tecnológicos para personas con discapacidad visual

Tema Objetivo

Taller 1

Conocer las principales características de los productos de

Productos de apoyo para personas con apoyo para las personas que presentan discapacidad visual.

condición de discapacidad

Taller 2

Características y aplicaciones del programa

lector de pantalla Jaws

Taller 3

Adaptación de materiales didácticos para las

personas con discapacidad visual
Conocer las principales características del lector de pantalla Jaws y sus aplicaciones en el sistema operativo Windows, Internet Explorer y Microsoft Office.

Diseñar materiales didácticos adaptados para este grupo de la diversidad.

Nota: Elaborado por Fontana y Rodríguez, 2012. Instrumentos del proyecto UNA Educación de Calidad.

Para llevar a cabo el objetivo del taller de la tabla 1, se proponen actividades de mediación que suscitaban la interacción entre los miembros del grupo; es decir, se tiene la oportunidad de realizar adaptaciones de materiales (impresiones en braille y en relieve), experimentar con los programas computación especializados con el fin de comprender su funcionamiento, particularmente con el lector de pantalla JAWS, asimismo de comprender la complejidad de los apoyos que requieren las personas con una condición de discapacidad visual en el contexto universitario. Además, cada taller cuenta con un instrumento de evaluación para recabar la opinión de los participantes de manera inmediata, para ir realizando los ajustes requeridos. 
URL: http://www.una.ac.cr/educare

CORREO: educare@una.cr

\section{Talleres de lectura y escritura en el sistema braille}

Los talleres para el aprendizaje de la lectura y escritura mediante el sistema braille constituyen un proceso construido y ejecutado por un estudiante HA con discapacidad visual de la carrera de Educación Especial con énfasis en Integración, quién contó con el apoyo de las académicas responsables para coordinar el proceso y orientar el componente pedagógico.

Estos talleres fueron ofrecidos durante el año 2010 por este estudiante a la comunidad estudiantil y se impartió en 8 sesiones de dos horas semanales cada uno, con partición de estudiantes de las carreras de Educación, Administración y Economía.

En la tabla 2 una descripción del segundo grupo de los talleres de este módulo.

Tabla 2

Tema y objetivo de los talleres de lectura y escritura del sistema braille

\begin{tabular}{ll}
\hline Tema & Objetivos \\
\hline $\begin{array}{l}\text { Taller } 1 \\
\text { Aprestamiento en el sistema braille }\end{array}$ & $\begin{array}{l}\text { Iniciar el proceso de acercamiento al sistema braille a través de diferentes } \\
\text { técnicas y actividades para concientizar a los estudiantes sobre los } \\
\text { elementos que lo componen. }\end{array}$ \\
\hline $\begin{array}{l}\text { Taller } 2 \\
\text { El alfabeto en braille }\end{array}$ & $\begin{array}{l}\text { Aprender las letras del abecedario utilizando el código generador del } \\
\text { sistema braille. }\end{array}$ \\
\hline $\begin{array}{l}\text { Taller } 3 \\
\text { El alfabeto en braille }\end{array}$ & $\begin{array}{l}\text { Aprender un nuevo grupo de letras del abecedario utilizando el código } \\
\text { generador del sistema braille. }\end{array}$ \\
\hline Taller 4 & $\begin{array}{l}\text { Aprender un nuevo grupo de letras del abecedario utilizando el código } \\
\text { El alfabeto en braille }\end{array}$ \\
\hline Taller 5 & generador del sistema braille. \\
Construyendo palabras & Iniciar el proceso de escritura de palabras utilizando las letras aprendidas. \\
\hline Taller 6 & Iniciar el proceso de escritura de palabras utilizando las letras \\
Escritura de palabras & aprendidas con el sistema braille. \\
\hline Taller 7 & Conocer los signos de puntuación y mayúsculas en el sistema de \\
Signos de puntuación & escritura braille. \\
\hline Taller 8 & Iniciar el proceso de escritura de oraciones en el sistema braille. \\
Construyendo oraciones & \\
\hline
\end{tabular}

Nota. Elaborado por Fontana y Rodríguez, 2012. Instrumentos del proyecto UNA Educación de Calidad.

Para desarrollar los talleres presentados en la tabla 2, se plantearon actividades de mediación que facilitaron un proceso gradual de identificación táctil de los signos que componen el sistema braille, hasta la construcción de palabras, frases y oraciones según su interés.

El proceso fue desarrollado de forma constructivista, ya que permitía aprender haciendo y experimentando. También, cada taller contaba con un instrumento de valoración que permitió 
conocer la opinión del grupo de participantes y realizar las adaptaciones correspondientes. Destaca la atención individual para cada participante.

\section{Talleres de autonomía y vida independiente}

Los talleres de autonomía y vida independiente fueron desarrollados y aplicados por un estudiante con discapacidad visual como parte de las actividades de curso de Intervención Pedagógica del Bachillerato en Educación Especial con énfasis Integración. Los talleres fueron ofrecidos a la comunidad estudiantil que participaba del proyecto en el año 2010. El propósito de estos fue potenciar las habilidades y destrezas personales del grupo de participantes en la dimensión personal y social.

Tabla 3

Tema y objetivo de los talleres de autodeterminación y vida independiente

\begin{tabular}{|c|c|}
\hline Tema & Objetivos \\
\hline $\begin{array}{l}\text { Taller } 1 \\
\text { Descubriendo mi autonomía e } \\
\text { independencia } \\
\text { en la vida universitaria }\end{array}$ & $\begin{array}{l}\text { Reconocer la importancia de la autonomía e independencia en la } \\
\text { formación universitaria y su impacto en la futura inserción laboral. }\end{array}$ \\
\hline $\begin{array}{l}\text { Taller } 2 \\
\text { Mis habilidades creativas }\end{array}$ & $\begin{array}{l}\text { Reconocer las diferentes manifestaciones de la diversidad personal y } \\
\text { social, y su impacto en el ambiente universitario. }\end{array}$ \\
\hline $\begin{array}{l}\text { Taller } 3 \\
\text { Me valoro, me aprecio y me cuido }\end{array}$ & $\begin{array}{l}\text { Comprender de qué forma se pueden potenciar las destrezas personales } \\
\text { en el contexto universitario, a través de la propia valoración. }\end{array}$ \\
\hline
\end{tabular}

Nota. Elaborado por Fontana y Rodríguez, 2012. Instrumentos del proyecto UNA Educación de Calidad.

Para alcanzar los objetivos de los talleres de la tabla 3, las actividades de mediación propuestas se enfocaron en desarrollar un proceso de reflexión personal sobre la importancia de la autonomía, especialmente en cuanto a la responsabilidad en la toma de decisiones en el ámbito universitario y su impacto en la formación profesional.

Las actividades fueron desarrolladas de forma constructivista, ya que permitía comprender, proponer y analizar situaciones cotidianas de la realidad educativa. También, cada taller contaba con su instrumento de valoración que facilitó conocer las observaciones del grupo de participantes.

\section{Taller de inducción al grupo de compañeros y compañeras de estudiantes con discapacidad visual}

El taller de inducción al grupo de compañeros y compañeras de estudiantes con discapacidad visual se ofrece a la comunidad estudiantil desde el año 2010, específicamente, se ha impartido en cursos de la carrea de Orientación, Pedagogía para I y II ciclos del Educación Básica, Geografía, Enseñanza de los Estudios Sociales y Cívica, Educación Especial con énfasis en Integración, Planificación y Promoción Social que tienen inscritos estudiantes con esta 
URL: http://www.una.ac.cr/educare

CORREO: educare@una.cr

condición. Estos talleres tienen el propósito de sensibilizar a la población estudiantil, asimismo, al personal académico promoviendo, de esta forma, la comprensión hacia la condición de discapacidad visual que presentan este grupo de la diversidad, con el fin establecer una relación de interacción y de apoyo de manera adecuada.

Seguidamente se presenta en la tabla 4 la descripción del cuarto grupo de talleres de este módulo.

Tabla 4

Tema y objetivo de taller de inducción al grupo de compañeros de curso de estudiantes con discapacidad visual

\begin{tabular}{ll}
\hline Tema & Objetivos \\
\hline Taller 1 & $\begin{array}{l}\text { Conocer el origen, las características y las formas de apoyo } \\
\text { Las personas con discapacidad visual y los que requieren las personas que presentan discapacidad } \\
\text { productos de apoyo en el contexto universitario } \\
\text { visual y sus productos de apoyo. }\end{array}$ \\
\hline $\begin{array}{l}\text { Taller } 2 \\
\text { Las personas con sordera y las formas de apoyo } \\
\text { en el contexto universitario }\end{array}$ & $\begin{array}{l}\text { Conocer el origen, las características y las formas de apoyo } \\
\text { que requieren las personas que presentan una condición } \\
\text { de sordera y las formas de apoyo. }\end{array}$ \\
\hline $\begin{array}{l}\text { Taller } 3 \\
\text { Las personas con discapacidad físico-motora y los } \\
\text { productos de apoyo en el contexto universitario }\end{array}$ & $\begin{array}{l}\text { Conocer el origen, las características y las formas de apoyo } \\
\text { qúsico- motora y sus productos de apoyo. }\end{array}$ \\
\hline
\end{tabular}

Nota. Elaborado por Fontana y Rodríguez, 2012. Instrumentos del proyecto UNA Educación de Calidad.

Las actividades propuestas para desarrollar los talleres mostrados en la tabla 4, promocionaron a las personas en condición de discapacidad, ya que estos se desarrollan considerando las capacidades y los recursos disponibles de cada individuo. Por ejemplo, se elaboraron materiales informativos según la causa de la deficiencia visual, auditiva o físicomotora, tales como retinosis pigmentaria, retinoblastoma, síndrome de Marfan, parálisis cerebral infantil y otras condiciones que presenta cada estudiante para informar al grupo de compañeros y compañeras. Asimismo, se realiza una demostración del uso adecuado de las ayudas técnicas como el bastón, el audífono, el programa lector de pantalla JAWS, el sistema de lectura y escritura en braille, el lenguaje de señas costarricense LESCO, entre otras.

Al presentar este taller se da una interacción constante entre el grupo de participantes, la conversación espontánea se convierte en una herramienta valiosa para recoger las apreciaciones del grupo de participantes y los aspectos por mejorar.

\section{Módulo 2: Talleres ofrecidos al grupo de estudiantes con discapacidad o necesidades educativas}

Del año 2010 al 2012 cinco estudiantes que realizaban HA en el proyecto desarrollaron y ejecutaron una serie de talleres dirigidos al grupo de estudiantes con discapacidad o necesidades 
educativas. Estas acciones fueron parte del proceso de culminación del proyecto de Práctica Profesional Supervisada en el Bachillerato en Orientación y en el Bachillerato en Educación Especial con énfasis en Integración; asimismo, del trabajo final de graduación en la Licenciatura de Educación Especial.

Estos talleres fueron construidos e impartidos con el propósito de satisfacer diferentes necesidades del grupo de estudiantes participantes en el proyecto en el ámbito personal académico y social.

A continuación se presenta en la tabla 5 el primer grupo de talleres de este módulo.

Tabla 5

Tema y objetivos de los talleres para potenciar las diversas formas de aprender, el uso de las técnicas de estudio y herramientas tecnológicas su formación profesional

\begin{tabular}{|c|c|}
\hline Tema & Objetivos \\
\hline $\begin{array}{l}\text { Taller } 1 \\
\text { Conociéndonos }\end{array}$ & $\begin{array}{l}\text { Conocer las características personales y académicas de la } \\
\text { población estudiantil que participa en los talleres. }\end{array}$ \\
\hline $\begin{array}{l}\text { Taller } 2 \\
\text { ¿Cómo aprendo? }\end{array}$ & $\begin{array}{l}\text { Identificar el estilo de aprendizaje predominante en cada } \\
\text { participante y las diferentes técnicas de estudio que emplean. }\end{array}$ \\
\hline $\begin{array}{l}\text { Taller } 3 \\
\text { Técnicas de estudio }\end{array}$ & $\begin{array}{l}\text { Desarrollar las principales características de algunas técnicas } \\
\text { de estudio con el fin de compararlas con el estilo de } \\
\text { aprendizaje que presenta cada participante. }\end{array}$ \\
\hline $\begin{array}{l}\text { Taller } 4 \\
\text { Conociendo mis hábitos de estudio y su } \\
\text { relación con el estilos de aprendizaje }\end{array}$ & $\begin{array}{l}\text { Conocer aspectos generales de la historia de vida en el ámbito } \\
\text { académico del grupo de participantes estableciendo una } \\
\text { relación con los hábitos de estudio que cada uno presenta. }\end{array}$ \\
\hline $\begin{array}{l}\text { Taller } 5 \\
\text { Mi radiografía }\end{array}$ & $\begin{array}{l}\text { Conocer mayor información de la historia de vida en el } \\
\text { ámbito académico del grupo de participantes. }\end{array}$ \\
\hline Talleres 6 y 7 Compartiendo experiencias & $\begin{array}{l}\text { Reflexionar sobre las experiencias personales y académicas } \\
\text { de cada participante. }\end{array}$ \\
\hline $\begin{array}{l}\text { Taller } 8 \\
\text { Involúcrame y lo aprendo }\end{array}$ & $\begin{array}{l}\text { Evocar los conocimientos adquiridos en los talleres anteriores } \\
\text { con el fin de relacionaran el estilo de aprendizaje que } \\
\text { presentan con las técnicas y condiciones reales de estudio. }\end{array}$ \\
\hline $\begin{array}{l}\text { Taller } 9 \\
\text { Reflexionando y proponiendo }\end{array}$ & $\begin{array}{l}\text { Reflexionar sobre la relación entre las técnicas de estudio, } \\
\text { el estilo de aprendizaje y las condiciones reales de cada } \\
\text { participante. }\end{array}$ \\
\hline $\begin{array}{l}\text { Taller } 10 \\
\text { Evaluación final y cierre de los talleres }\end{array}$ & $\begin{array}{l}\text { Evaluar el proceso vivenciado a lo largo del desarrollo de } \\
\text { los talleres. }\end{array}$ \\
\hline
\end{tabular}

Nota: Elaborado por Fontana y Rodríguez, 2012. Instrumentos de Arce y Ávila (2011) proyecto UNA Educación de Calidad. 
URL: http://www.una.ac.cr/educare

CORREO: educare@una.cr

La aplicación de los talleres en la tabla 5 permitió recabar información para elaborar una caracterización del grupo de participantes en cuanto a su estilo de aprendizaje y se constituyó en el referente primordial para la elaboración y adaptación de los talleres, técnicas de estudio y el uso de herramientas tecnológicas (programas computacionales y otros). Además, se tomaron en consideración las observaciones y apreciaciones aportadas en el buzón de sugerencias para valorar el impacto en el área académica y en el diseño de la propuesta final.

Seguidamente se presenta en la tabla 6 la descripción del segundo grupo de talleres de este módulo.

Tabla 6

Tema y objetivos de los talleres de desarrollo personal en el ámbito universitario

\begin{tabular}{|c|c|}
\hline Tema & Objetivos \\
\hline $\begin{array}{l}\text { Taller } 1 \\
\text { La sexualidad, los mitos y los estereotipos }\end{array}$ & $\begin{array}{l}\text { Generar un acercamiento de la sexualidad } \\
\text { humana, sus mitos y estereotipos de una manera } \\
\text { integral mediante la reflexión y la discusión sobre } \\
\text { experiencias personales. }\end{array}$ \\
\hline $\begin{array}{l}\text { Taller } 2 \\
\text { Mi autoestima y los factores internos y externos que la } \\
\text { determinan }\end{array}$ & $\begin{array}{l}\text { Reflexionar sobre la autoestima que presenta cada } \\
\text { estudiante y los factores externos e internos que la } \\
\text { determinan. }\end{array}$ \\
\hline $\begin{array}{l}\text { Taller } 3 \\
\text { La comunicación asertiva y la expresión de sentimientos }\end{array}$ & $\begin{array}{l}\text { Desarrollar una comunicación asertiva y expresión } \\
\text { de sentimientos con el fin de adquirir las } \\
\text { herramientas necesarias para la comunicación con } \\
\text { personas dentro y fuera del ambiente universitario. }\end{array}$ \\
\hline $\begin{array}{l}\text { Taller } 4 \\
\text { Mis relaciones interpersonales }\end{array}$ & $\begin{array}{l}\text { Desarrollar habilidades sociales que les permitan } \\
\text { interrelacionarse con las demás personas de } \\
\text { manera apropiada. }\end{array}$ \\
\hline $\begin{array}{l}\text { Taller } 5 \\
\text { Manejando del estrés }\end{array}$ & $\begin{array}{l}\text { Desarrollar técnicas para el manejo del estrés en } \\
\text { el ámbito personal y académico, por medio de la } \\
\text { obtención de conocimientos teóricos y vivenciales, } \\
\text { para que puedan enfrentar las tensiones de sus } \\
\text { actividades diarias. }\end{array}$ \\
\hline $\begin{array}{l}\text { Taller } 6 \\
\text { Compartiendo experiencias }\end{array}$ & $\begin{array}{l}\text { Evaluar el desarrollo de los talleres mediante la } \\
\text { reflexión y retroalimentación en los participantes, } \\
\text { con el fin de que compartan la experiencia vivida y } \\
\text { el conocimiento adquirido. }\end{array}$ \\
\hline
\end{tabular}

Nota. Elaborado por Fontana y Rodríguez, 2012. Instrumentos del proyecto UNA Educación de Calidad. 
Las actividades propuestas en los talleres de la tabla 6 facilitaron al grupo de participantes con discapacidad o necesidades educativas contar con un espacio para compartir sobre sus experiencias personales en el proceso universitario, identificar aspectos de su vida que requerían potenciar, o bien que limitan su desarrollo personal y académico.

Estos talleres siguieron una lógica participativa de construcción de las temáticas de interés por parte del grupo de participantes y de intercambio constante de experiencias en un marco de respeto y aceptación de la diversidad personal de cada uno. Además, el último taller se destinó para valorar la dinámica desarrollada en los talleres, insumo valioso para futuras acciones.

\section{Módulo 3: Talleres ofrecidos para el grupo de estudiantes que realizan labores de colaboración}

Debido a las características del trabajo que despliega el proyecto UNA Educación de Calidad, se recibe, por ciclo lectivo, el apoyo de un grupo aproximado de 30 estudiantes de diferentes carreras que realizan $\mathrm{HC}$ y $\mathrm{HA}$.

Esta modalidad de asistencia a los programas, proyectos y actividades académicas que se ejecuten en el ámbito universitario está establecida en el Manual de organización de la Vicerrectoría de Vida Estudiantil (VVE) de la UNA, en el capítulo De los fundamentos del sistema de becas y su organización, como un principio de colaboración de la población estudiantil que recibe una beca en la universidad, por considerarse importante su participación en los proyectos, programas y actividades académicas para su desarrollo personal y profesional (Universidad Nacional, 2012).

El apoyo de este grupo de estudiantes es fundamental para el quehacer del proyecto, particularmente, para dar atención directa al grupo de estudiantes con discapacidad o que presenta necesidades educativas.

Entre las principales formas de apoyo, se destaca el trabajo que realizan con sus pares que presentan una condición de discapacidad visual, tales como la lectura oral o el grabado de textos, documentos o libros requeridos en los cursos matriculados; la orientación y la movilidad dentro del contexto universitario, cuando es solicitada; la digitalización de los documentos escritos; la adaptación de los materiales y recursos (impresiones en el sistema braille, ampliaciones o en relieve); el apoyo como referente visual en el salón de clase y en la visita a diferentes lugares fuera de la institución, entre otras acciones.

Durante el 2008 y 2010 se realizaron diferentes talleres para el grupo de estudiantes que realizan $\mathrm{HC}$, con el objetivo de promover un espacio de reflexión sobre las experiencias que vivencian en la dinámica del proyecto y generar nuevos aprendizajes en relación con el apoyo brindado al grupo de estudiantes con discapacidad o necesidades educativas. 
URL: http://www.una.ac.cr/educare

CORREO: educare@una.cr

Seguidamente se presenta, en la tabla 7, el tercer grupo de talleres de esta modalidad.

Tabla 7

Tema y objetivos del taller sobre las formas de apoyo del grupo de estudiantes que realizan labores de colaboración en el Proyecto UNA Educación de Calidad

\begin{tabular}{ll}
\hline Tema & Objetivos \\
\hline $\begin{array}{l}\text { Taller } 1 \\
\text { Mi experiencia de apoyo }\end{array}$ & $\begin{array}{l}\text { Describir el apoyo que brinda el grupo de estudiantes que realizan HC, } \\
\text { con los estudiantes con necesidades educativas. }\end{array}$ \\
& $\begin{array}{l}\text { Identificar las limitaciones y obstáculos que se han presentado durante } \\
\text { el cumplimiento de las horas colaboración. }\end{array}$ \\
& Identificar los nuevos aprendizajes construidos durante el apoyo al \\
& grupo de estudiantes con necesidades educativas. \\
\hline Taller 2 & $\begin{array}{l}\text { Identificar los sentimientos y emociones generados a partir del apoyo } \\
\text { Mis sentimientos y emociones }\end{array}$ \\
\hline
\end{tabular}

Nota. Elaborado por Fontana y Rodríguez, 2012. Instrumentos del proyecto UNA Educación de Calidad.

Para alcanzar los objetivos propuestos, se realizó, en el desarrollo de los talleres de la tabla 7, una combinación de actividades tanto en forma individual como grupal. También, se realizaron actividades de evaluación $y$, de las recomendaciones que emergieron en estos talleres, se aportó significativamente para mejorar y articular las labores que desempeña el grupo de estudiantes HC.

A continuación en la tabla 8 se presenta el último grupo de talleres de este módulo.

Tabla 8

Tema y objetivos del taller sobre las formas de apoyo del grupo de estudiantes que realizan labores de colaboración en el Proyecto UNA Educación de Calidad

\begin{tabular}{ll}
\hline Tema & Objetivos \\
\hline Taller 1 & $\begin{array}{l}\text { Describir las funciones y las responsabilidades de } \\
\text { este grupo de estudiantes que realiza HC, según la } \\
\text { Funciones de un estudiante que realiza HC de discapacidad. }\end{array}$ \\
\hline Taller 2 & $\begin{array}{l}\text { Determinar los logros alcanzados y las limitaciones } \\
\text { en su labor de apoyo. }\end{array}$ \\
\hline Logro y limitaciones & $\begin{array}{l}\text { Conocer las recomendaciones para mejorar su labor } \\
\text { de apoyo, colaboración y el seguimiento al grupo de } 3\end{array}$ \\
Aspectos por mejorar & estudiantes que realizan HC. \\
\hline
\end{tabular}

Nota. Elaborado por Fontana y Rodríguez, 2012. Instrumentos del proyecto UNA Educación de Calidad. 
En los talleres mencionados en la tabla 8, la estrategia desplegada facilita la discusión entre el grupo de participantes sobre la temática abordada, asimismo el aporte individual cuando se requería. Esto permitió recabar información sobre los logros alcanzados y las limitaciones tanto de experiencias individuales como de situaciones de grupo, según los apoyos brindados a una condición de discapacidad. Además, la evaluación se realizó en dos direcciones, una que registraba en cada taller las apreciaciones del grupo de participantes y la otra, al finalizar, mediante un instrumento que valoraba las responsabilidades correspondientes al equipo del proyecto.

\section{Resultados de los talleres}

La modalidad de los talleres es una estrategia eficaz para vincular estudiantes a la dinámica del proyecto UNA Educación de Calidad, tanto al grupo que realiza HC y HA como al grupo que recibe este apoyo; ya que les da una voz para proponer, coordinar, planear y ejecutar actividades desde sus habilidades y destrezas personales y sociales. Para promover una mejor comprensión, la reflexión general de los resultados se presenta según los módulos a los que se refieren. En cada uno de los talleres se diseñaron instrumentos de evaluación que permitieron recopilar la información para la construcción de los resultados, los cuales consistían en una guía de tres preguntas (abiertas) sobre el conocimiento construido, su aplicación en la vida personal y social, y el aporte de sugerencias. Posteriormente, las evaluaciones se digitalizaron en matrices para su análisis y discusión.

\section{Módulo 1. Talleres ofrecidos por estudiantes con discapacidad visual a la comunidad universitaria}

El proceso de construcción y ejecución de los talleres sobre tecnologías de apoyo ha sido positivo para el grupo de estudiantes que presenta una condición de discapacidad visual que participa del proyecto. Este espacio les ha permitido potenciar diversas capacidades en el ámbito personal y social, especialmente en el académico; ya que les ofrece una oportunidad de compartir sus conocimientos con la comunidad estudiantil a la cual pertenecen. Además, es importante resaltar que tanto la temática de los talleres como el proceso que se vive en su implementación, actualmente, es responsabilidad de los estudiantes con discapacidad visual: Sin duda se potencian sus capacidades para resolver conflictos y proponer soluciones, sus habilidades comunicativas y su creatividad, porque cada año que se lleva acabo se incorporan nueva actividades.

A su vez, los talleres permiten al grupo de participantes aprender sobre el uso de la tecnología para apoyar a las personas con discapacidad visual; esto, a través del conocimiento del programa lector de pantalla JAWS, el traductor a braille DBT Win, el diseñador de gráficos Quick Tack, así como el uso de equipo para realizar impresiones en el sistema braille y materiales en relieve con el horno Thermofon. 
URL: http://www.una.ac.cr/educare

CORREO: educare@una.cr

El grupo de estudiantes participantes manifestó que la metodología utilizada en los talleres era constructivista y el aprendizaje obtenido fue significativo para su formación académica y profesional, especialmente el uso del equipo y programas especializados.

Un aspecto que destaca todo el grupo involucrado es la importancia de comprender la necesidad de realizar ajustes y adaptaciones a los elementos del entorno físico y social, para que las personas con discapacidad puedan acceder a la educación en igualdad y equidad de condiciones.

Además, el grupo de participantes que cursaba carreras relacionadas con la pedagogía, mencionan que el conocimiento adquirido les ha permitido sensibilizarse y reconocer la complejidad de los ajustes que se requieren para abordar las necesidades educativas en los futuros contextos de trabajo.

En relación con los talleres impartidos sobre la lectura y escritura con el sistema braille, se destaca que el grupo de participantes logró adquirir el conocimiento básico de la simbología estudiada en los dos primeros meses, porque se constaba con el material requerido (álbum de aprestamiento, signo generador en madera, palitos de metal, pañuelos, regletas, punzón, abecedario y otros materiales impresos y en relieve) y la sesiones de práctica se dieron en forma gradual, rescatando los progresos individuales. Lo anterior permitió que el grupo de participantes desarrollara un proceso de lectura y escritura de acuerdo con sus capacidades e intereses, por lo cual esta resultó una experiencia significativa enriquecedora de su formación académica, especialmente al grupo que estudiaba pedagogía, porque adquirió habilidades técnicas para trabajar con las futuras poblaciones con discapacidad visual incluidas en los servicios de la educación general básica de Costa Rica.

Los talleres de auto determinación y vida independiente le permitieron, al estudiantado con discapacidad visual, apoyar a sus compañeros y compañeras desde sus intereses y habilidades. Por otra parte, el grupo de estudiantes que participaron de los talleres se mostró interesado en las temáticas y aportaron desde sus experiencias de vida y sobre las barreras que enfrentan en el contexto universitario.

Entre los aspectos por mejorar en esta modalidad de talleres se menciona que el acceso a los equipos, programas de computación y materiales especializados es fundamental para mantener el conocimiento adquirido, por lo que se considera de vital importancia el establecimiento de un horario en la oficina del proyecto UNA Educación de Calidad para que el grupo de participantes pueda realizar prácticas o bien crear materiales según su formación profesional.

\section{Módulo 2. Talleres ofrecidos al grupo de estudiantes con discapacidad o necesidades educativas}

Los talleres de desarrollo personal, autodeterminación y vida independiente le permitieron al grupo de estudiantes con discapacidad o necesidades educativas reflexionar sobre las áreas 
que requieren potenciar en sus vidas, tales como la comunicación asertiva, la comprensión de sus sentimientos y emociones; asimismo la utilización de diferentes técnicas personales de autocontrol, la identificación de sus derechos y las responsabilidades en el contexto personal, académico y social.

Un aspecto que se distingue entre los resultados es que el grupo de participantes indica que los talleres les permitieron identificar los aspectos individuales que requieren para desempeñarse y ajustarse mejor a sus procesos educativos en el ámbito universitario.

Por otro lado, el grupo de estudiantes participantes mencionó que existen brechas para hacer efectiva la inclusión social, ya que en reiteradas ocasiones los compañeros y las compañeras y el personal académico y administrativo, manifiestan estereotipos y prejuicios que desvalorizan los esfuerzos que realizan las personas con discapacidad o que presentan necesidades educativas. Reconocen que estas situaciones han ocasionado, en algunos casos, el aislamiento de las dinámicas sociales que se originan en sus cursos.

Por otra parte, la experiencia de los talleres de desarrollo personal les permitió expresar estos sentimientos, reconocer en sus compañeros y compañeras del proyecto un apoyo ante situaciones personales de este tipo $y$, finalmente, buscar alternativas para luchar de forma coordinada antes las situaciones que consideren discriminatorias.

Con relación con los talleres para potenciar las diversas formas de aprender, las técnicas de estudio y el uso de herramientas tecnológicas constituyeron, para el grupo de participantes, un espacio para visualizarse de forma integral, ya que se reflexionó sobre la formación del autoconcepto, su imagen personal, las habilidades y destrezas, asimismo acerca de las necesidades educativas.

Las actividades propuestas en estos talleres permitieron un reconocimiento del estilo, el ritmo y la forma personal para aprender de cada participante, donde el estilo visual y auditivo resultaron los predominantes, según la teoría neurolingüística del aprendizaje. Pero ningún miembro del grupo menciona alguna técnicas de estudio, las condiciones ambientales, los recursos materiales y tecnológicos que pueden emplear durante el periodo de estudio que favorezcan su desempeño en el ámbito académico, de acuerdo con sus características personales y sociales y las exigencias de la carrera que cursan.

No obstante, mediante el trabajo colaborativo, el grupo de estudiantes logró identificar las técnicas de estudio más apropiadas según su forma personal de aprender, estableciendo que esta herramienta involucra dos o más áreas sensoriales (visual, auditivo y kinestésico) del aprendizaje, lo cual favorecerá su aprovechamiento en diferentes ámbitos, especialmente, en el área académica.

También, el grupo de estudiantes alcanzó a identificar los procesos cognitivos que se involucran en las diferentes formas de aprender (la asociación, la clasificación, el análisis, la síntesis, la generalización, entre otros), las cuales son esenciales para un aprendizaje significativo en su formación profesional. 
URL: http://www.una.ac.cr/educare

CORREO: educare@una.cr

Por tanto, se concluye que la implementación de las técnicas de estudio por parte del grupo de estudiantes en su formación académica dependerá del conocimiento de los procesos cognitivos que se involucran en el aprendizaje, y del interés, la motivación, la persistencia, la seguridad personal de cada uno por ajustar las técnicas de estudio seleccionadas a sus características personales y sociales, a las condiciones ambientales, a los recursos materiales y tecnológicos que disponen. Los aspectos emocionales y metacognitivos se reconocieron como esenciales para la autodeterminación e independencia, así como para alcanzar un aprendizaje significativo en su formación profesional.

\section{Módulo 3. Talleres ofrecidos para el grupo de estudiantes que realizan labores de colaboración}

En general, el estudiantado que realiza $\mathrm{HC}$ se ha sensibilizado sobre los retos que enfrenta el grupo de estudiantes con discapacidad o que presentan necesidades educativas en el contexto universitario. En los talleres efectuados manifestó haber comprendido que todos poseen como estudiantes los mismos derechos y deberes. Sin embargo, reconocen la necesidad de realizar ajustes y adaptaciones a los elementos del entorno físico y social para que puedan disfrutar de los bienes y servicios de la sociedad; pero principalmente para generar las condiciones de accesibilidad que le permitan, a este grupo de la diversidad, acceder a una educación de calidad.

A nivel personal, en el grupo de estudiantes que realizan $\mathrm{HC}$, existe un sentimiento de satisfacción por las labores realizadas y menciona haber comprendido que la discapacidad no es un obstáculo para la vida, sino una oportunidad de ser mejores.

Durante el proceso de trabajo conjunto han aprendido a conocer a sus compañeros y compañeras con discapacidad o que presentan necesidades educativas y a respetarles como personas. En muchas ocasiones, el apoyo que se proporciona termina convirtiéndose en una amistad entre estos estudiantes, la cual se extiende más allá de la dinámica del proyecto y se proyecta hasta el ámbito social.

Los diferentes espacios de interacción e intercambio entre el grupo estudiantes que realizan $\mathrm{HC}$ y el grupo de estudiantes que participan del proyecto, particularmente, en condición de discapacidad, han permitido una reconstrucción de la visión tradicional sobre la personas con discapacidad, logrando posicionarse en una perspectiva centrada en las cualidades y talentos. Esto les ha permitido desenvolverse en forma exitosa en diferentes contextos sociales y alcanzar sus metas.

Los sentimientos de incertidumbre sobre el trato que se les debe proporcionar a las personas con una condición de discapacidad, por parte del grupo de estudiantes que realizan $\mathrm{HC}$ en el proyecto, pone en evidencia que la historia de segregación y diferenciación social en la atención educativa, en la cual han estado inmersos ambos grupos, limita la adquisición de las destrezas básicas para establecer relaciones interpersonales en forma adecuada. 
Un aspecto que destaca el grupo que efectúa HC es el papel fundamental del estudiante con discapacidad, porque es la persona responsable no solo de velar el cumplimiento de sus derechos según la legislación nacional vigente, sino de promover una concienciación entre los miembros de la comunidad universitaria sobre el valor de diversidad y su inclusión social.

Para mejorar las labores de colaboración, estos estudiantes recomiendan, al equipo del proyecto, desarrollar material de apoyo que les permita comprender sus responsabilidades y funciones como estudiantes que realizan $\mathrm{HC}$; además, recomendaron continuar realizando talleres que les permita tener estos espacios de reflexión para compartir sus vivencias en el contexto universitario.

\section{Conclusiones}

A la luz de las experiencias en los diferentes talleres en el contexto del proyecto UNA Educación de Calidad se puede concluir que:

- Los talleres son una estrategia novedosa que permite el aprendizaje colaborativo desde la mirada de múltiples actores que conforman el proyecto UNA Educación de Calidad, especialmente, se destaca, en los diferentes módulos construidos, el establecimiento de roles según las capacidades y una interrelación simétrica entre el conglomerado, lo cual convierte este espacio en una oportunidad para un crecimiento personal, académico y social.

- Debido a su carácter participativo, la modalidad de los talleres permite el intercambio de ideas, la reflexión y la reconstrucción de las temáticas abordadas desde las realidades de quienes participan, facilitando, de esta forma, la satisfacción de las necesidades inmediatas, el aporte de alternativas creativas y solidarias, la capacidad de resiliencia y de trabajo en equipo, las diversas formas de comunicación y negociación en situaciones divergentes; aspectos fundamentales para una adecuada convivencia en el contexto universitario y su formación profesional.

- Los diferentes talleres desplegados han sido una herramienta muy importante en el quehacer del proyecto UNA Educación de Calidad, principalmente para empoderar al grupo de estudiantes con discapacidad visual. Este grupo de estudiantes ha planificado y ejecutado acciones de colaboración utilizando sus conocimientos y destrezas desarrolladas desde su condición de discapacidad, para que sus pares en el contexto universitario se sensibilicen y comprendan la imperante necesidad de generar las condiciones de accesibilidad para que puedan acceder a la educación en igualdad y equidad, según su diversidad personal y social.

- Un aspecto que se distingue es que la modalidad de los talleres ha permitido a la comunidad universitaria, particularmente a la población estudiantil, un acercamiento al quehacer del proyecto, al facilitar la comprensión de la complejidad de los apoyos y los múltiples servicios que ofrece; por tanto, este espacio realiza un aporte social a la comunidad nacional. 
URL: http://www.una.ac.cr/educare

CORREO: educare@una.cr

- La experiencia de los talleres y los resultados significativos obtenidos instan al equipo del proyecto UNA Educación de Calidad a continuar con la metodología desplegada desde un enfoque constructivista, que les permita a sus participantes continuar colaborando, desde sus ámbitos de formación.

\section{Consideraciones finales}

La cotidianidad del quehacer del proyecto UNA Educación de Calidad en la Universidad Nacional es sumamente compleja, debido a que aproxima la colaboración de múltiples actores que, desde sus posibilidades, habilidades y destrezas, ofrecen al proyecto lo mejor de sí, conformando una gran red de apoyo que se acrecienta con el paso de los años.

En esta red de apoyo, el grupo de estudiantes que realiza labores de asistencia y colaboración es muy significativo, ya que ellos y ellas contribuyen día con día en el trabajo que se requiere realizar y ofrecen, al grupo de estudiantes con discapacidad o necesidades educativas, novedosas experiencias sociales y educativas que les permiten aprender de forma conjunta.

Sin embargo, la complejidad va más allá, al integrar, en el quehacer cotidiano del proyecto, las múltiples experiencias de los estudiantes con una condición de discapacidad o necesidades educativas, quienes aportan aprendizajes significativos, desde su historia de vida, a las discusiones que se realizan en términos de respeto, igualdad y equidad a los derechos individuales y sociales, así como a los deberes y responsabilidades académicas.

A su vez, la comunidad estudiantil participante en los talleres se transforma al conocer y reconocer los esfuerzos, destrezas y apoyos que requiere la población que participa del proyecto. Al final, las académicas que colaboramos como responsables del proyecto UNA Educación de Calidad somos, simplemente, quienes orquestamos todas las voces, ideas y experiencias de los muchos estudiantes que desean trabajar por el bienestar y desarrollo de la comunidad educativa de la Universidad Nacional con discapacidad o necesidades educativas, en un marco de respeto, autogestión y vida independiente.

\section{Referencias}

Abad, M., Álvarez, P. y Castro, J. F. (2008). Apoyo a la integración de estudiantes con discapacidad en la enseñanza universitaria: Algunas medidas y propuestas de actuación orientadora. Educación y Diversidad, 2, 129-150. Recuperado de http://dialnet.unirioja.es/servlet/ articulo?codigo $=2547369$

Arce, J. y Ávila, L. (2011). Propuesta de apoyo educativo para los y las estudiantes que participan del Proyecto UNA Educación de Calidad de la Universidad Nacional con el fin de potenciar las diversas formas de aprender y desplegar nuevas destrezas y habilidades personales, 
académicas y sociales en su formación universitaria (Tesis de Licenciatura inédita). Universidad Nacional, Heredia, Costa Rica.

Candelo, C., Ortiz, G. y Unger, B. (2003). Hacer talleres: Una guía práctica para capacitadores. WWF Cali: Grafiq editores. Recuperado de http://www.gwp.org/Global/GWP-SAm Files/ Publicaciones/Hacer-talleres-gu\%C3\%ADa-para-capacitadores-esp.pdf

Consejo Nacional de Rehabilitación y Educación Especial (CNREE). (2011). Política Nacional en Discapacidad 2011-2021. San José, Costa Rica: Autor. Recuperado de http://www.cnree. go.cr/images/stories/Documentos/Ponadis.pdf

Costa Rica, Asamblea Legislativa. (29 de mayo de 1996). Igualdad de oportunidades para las personas con discapacidad (Ley N 7600). La Gaceta. Diario Oficial N 102. San José, Costa Rica: Imprenta Nacional.

Costa Rica, Asamblea Legislativa. (29 de setiembre del 2008). Convención sobre los derechos humanos de las personas con discapacidad (Ley N 8661). La Gaceta. Diario Oficial N 187. San José: Imprenta Nacional.

Cortes, M. J. e Iriarte, G. (s. f.). El taller. Modelo para una metodología globalizada e interdisciplinar en la unidad de currículo especial. Recuperdo de http://centros.educacion.navarra.es/ creena/008psiquicos/PDFs/4.\%20EL\%20TALLER.\%20Modelo\%20para\%20una\%20 $\underline{\text { metodologia.pdf }}$

Echeita, G. y Martín, E. (1999). Interacción social y aprendizaje. En C. Coll, J. Palacios y Marchesi, A. (Comps.), Desarrollo psicológico y educación. (Vol. 1, pp. 49-67). Madrid: Alianza Editorial.

Fontana, A. (diciembre, 2009). Retos de la atención a la diversidad: El caso de la Universidad Nacional de Costa Rica. Visón Docente Con-Ciencia, 9(51), 30-39. Recuperado de http://www. ceuarkos.com/Vision docente/revistas/51/RETOS.pdf

Fontana, A. y Rodríguez, R. (2011). Formulación de la ll fase del proyecto UNA educación de calidad 2012-2014 (código 0499-11). Heredia: Vicerrectoría Académica, Universidad Nacional. Manuscrito inédito.

Fontana, A. y Rodríguez, R. (2012). Informe anual del proyecto UNA educación de calidad (código 0499-11). Heredia: Vicerrectoría Académica, Universidad Nacional. Manuscrito inédito.

Hernández, R., Fernández, C. y Batista P. (2006). Metodología de la investigación. México D. F.: McGraw-Hill.

Maldonado, M. y Sánchez, T. (mayo-agosto, 2012). Trabajo colaborativo en el aula: Experiencias desde la formación docente. Educare, 16(2), 93-118. Recuperado de http://revistas.upel.edu. ve/index.php/educare/article/view/822 
URL: http://www.una.ac.cr/educare

CORREO: educare@una.cr

Organización de las Naciones Unidas [ONU] (2006). Convención sobre los derechos de las personas con discapacidad. Recuperado de http://www.un.org/spanish/disabilities/documents/gid/ conventionfaq.pdf

Universidad Nacional (UNA). (1993). Estatuto Orgánico. Heredia: Departamento de Publicaciones UNA. Recuperado de http://www.juridica.una.ac.cr/index.php?option=com remository\&lt emid $=0 \&$ func $=$ startdown\&id $=144$

Universidad Nacional (UNA). (15 de febrero del 2012). Manual de organización y funciones de la Vicerrectoría de Vida Estudiantil. UNA-Gaceta No 2. Recuperado de http://www.juridica.una. ac.cr/index.php?option=com remository\&ltemid $=0 \&$ func $=$ startdown\&id $=384$

\section{Cómo citar este artículo en APA:}

Rodríguez, M. R. y Fontana, A. (enero-abril, 2014). La estrategia de trabajo colaborativo del Proyecto UNA Educación de Calidad de la Universidad Nacional de Costa Rica: Experiencia de la modalidad de talleres Revista Electrónica Educare, 18(1), 193-218. Recuperado de http://www.revistas.una.ac.cr/index.php/EDUCARE/issue/current

Nota: Para citar este artículo en otros sistemas puede consultar el hipervínculo "Como citar el artículo" en la barra derecha de nuestro sitio web:

http://www.revistas.una.ac.cr/index.php/EDUCARE/index 